\title{
Applicability of the Resistance Gene- Like Fragment ADG2 as an RFLP Probe in Selection of Extreme Resistance to Potato Y Potyvirus (PVY)
}

\author{
Akio SHIRANITA*, Kazue KASAI*, Jaana H. HÄMÄLÄINEN**, Jari P. T. VALKONEN**, \\ and Kazuo N. WATANABE*1) \\ *Department of Biotechnological Science, Kinki University, Uchita, Wakayama, \\ 649-6493, Japan \\ **Genetic Centre, Swedish University of Agricultural Sciences (SLU), P.O. Box 7025, \\ S-75007 Uppsala, Sweden \\ ${ }^{1)}$ Corresponding author $\mathrm{E}$-mail:
}

Received 9 February 1999; accepted 24 August 1999

\begin{abstract}
Resistance gene-like fragment ADG2 has been shown to co-segregate with $R y_{\text {adg }}$, which controls extreme resistance to potato Y potyvirus (PVY) in Solanum tuberosum subsp. andigena (Hämäläinen et al. 1997, 1998). Applicability tests of ADG2 as a selection tool for resistance to PVY were carried out by genomic Southern analysis on a broad range of cultivars and breeding lines of potato and some other solanaceous species. A new marker band $(10.5 \mathrm{~kb})$ associating with $R y$ was found in addition to previously reported $3.5 \mathrm{~kb}$ marker band. While the $3.5 \mathrm{~kb}$ marker was specifically linked to $R y_{a d g}$ with extremely high association ( $96 \%$ ), the newly identified $10.5 \mathrm{~kb}$ marker band was detected in genotypes containing $R y_{a d g}$ and other $R y$ genes. Some signals with ADG2 were also observed in other species within family Solanaceae inferring with the diversity of the corresponding chromosome region(s) among solanaceous species.
\end{abstract}

\section{Introduction}

PVY is a member of the genus Potyvirus which contains ca. 180 of the total of 200 virus species belonging to the family Potyviridae that, in turn, contains ca. $25 \%$ of all known plant viruses (Shukla et al. 1994). PVY is transmitted by aphids in the field in a non-persistent manner and naturally infects potato, pepper, tomato, and other solanaceous plant species (de Bokx and Huttinga 1981). PVY is characterized by occurrence under an extremely wide range of environmental conditions.

Globally, potato is the fourth most important food crop after wheat, corn and rice (Hawkes 1990). In Northern Europe, Russia, North and South America and Japan, PVY is a particularly important viral pathogen of cultivated potatoes and causes serious problems for potato production as it can reduce yields up to $80 \%$ (Hooker et al. 1981).

Potato viruses such as PVY are transmitted to succeeding potato generations through tubers (Valkonen et al. 1996). Therefore, the important methods for control of PVY in potato crops include the use of virus - free seed potatoes and pesticides that kill virus vectors (de Bokx and van der Want 1987). However, there are some problems as pesticides have harmful effects on environment. Despite these control efforts, susceptible cultivars become infected with PVY in the field, and the cost of renewing virus -free seed potatoes can not be avoided.

Potato cultivars with the resistance to viruses are the best option for virus control. However, development of resistant cultivars has been difficult because the tetraploid potatoes $(2 n=4 x=48)$ show a complicated inheritance of traits and breeding has consequently required considerable labor and time. Marker-assisted selection (MAS) for resistance can alleviate these problems and improve the cost effectiveness by speeding up the introgression of resistant genes (Watanabe et al. 1994a, 1995a).

Two major types of monogenically inherited resistance to potato $\mathrm{Y}$ potyvirus (PVY) are known in cultivated and wild potato species (Solanum spp.), namely extreme resistance (E) and hypersensitive resistance $(\mathrm{H})$. E is controlled by $R y$ genes and are effective against all strains of PVY, whereas $\mathrm{H}$ controlled by $N y$ genes is often PVY strain groupspecific. Following infection with PVY, potato 
plants expressing $\mathrm{Ny}$ develop necrotic lesions in infected leaves and/or necrosis in systemically infected parts, whereas the plants expressing $R y$ remain symptomless, except that limited necrosis may develop in the systemically infected leaves in a few genotypes following graft-inoculation, and no PVY titers detectable with ELISA develop in inoculated plants expressing Ry (Cockerham 1970, Ross 1986, Jones 1990, Valkonen et al. 1996). The expression of $R y$ is epistatic to the expression of $N y$ (Valkonen et al. 1994a).

The gene $R y_{a d g}$ controls $\mathrm{E}$ to PVY in the cultivated potato $S$. tuberosum subsp. andigena and is located on chromosome XI (Hämäläinen et al. 1997, 1998). Another $R y$ gene is also located at the close position on chromosome XI (Brigneti et al. 1997) as are many additional disease resistance genes in other potato species and in Nicotiana species (reviewed by Hämäläinen et al. 1998).

Up to now some RFLP markers that co-segregate with $R y_{a d g}$ have been identified (Hämäläinen $e t$ al. 1997, 1998). Also PCR - based markers such as CAPS (Cleaved Amplified Polymorphic Sequences) and SCAR (Sequence Characterized Amplified Regions) are available (Sorri et al. 1999, Kasai et al. 1999). However, these markers are considered $R y_{a d g}$ -specific.

A DNA fragment designated as ADG2 has been previously amplified from $S$. tuberosum subsp. andigena with primers that are designed according to highly similar regions at isolated disease resistance genes (Leister et al. 1996, Hämäläinen et al. 1998, Sorri et al. 1999, Kasai et al. 1999). This fragment co-segregates with $R y_{a d g}$ among the 77 progeny without recombination and, thus, identifies the locus on chromosome XI that is known to contain resistance clusters in many species of Solanaceae (Leister et al. 1996, Hämäläinen et al. 1998).

Although DNA fragment ADG2 can be amplified from PVY susceptible potato genotypes, twelve nucleotide change was found between ADG2 fragments of PVY resistant $2 \times(V-2)_{7}$ and PVY susceptible 84.194.30. ADG2 fragment from $2 \times(\mathrm{V}-2)_{7}$, which contain $R y_{a d g}$, shows $77 \%$ nucleotide sequence homology with the corresponding region of the gene $N$ for resistance to tobacco mosaic virus in Nicotiana glutinosa, and $53 \%$ homology with RPP5 for resistance to Peronospora parasitica in Arabidopsis thaliana (Hämäläinen et al. 1998, Sorri et al. 1999). Moreover, two out of twelve nucleotide differences were located in predicted kinase -2 and kinase -3a motif causing deduced amino acid changes (Sorri et al. 1999). Taking these findings into consideration, it has been suggested that ADG2 fragment may be parts of $R y_{a d g}$. Therefore, it is meaningful to search for homologues of the ADG2 fragment in other solanuceous species to associate with the potential existence of a resistance gene (Watterson et al. 1993).

In this study, applicability of MAS for resistance to PVY using ADG2 as an RFLP probe was tested on a genetically diverse range of the potato cultivars and breeding lines, and also other solanaceous crop species that are affected by PVY.

\section{Materials and Methods}

\subsection{Plant materials}

A total of 117 diploid and tetraploid potato breeding lines; and diploid, tetraploid, and pentaploid potato cultivars with various genetic backgrounds (Table 1), were tested with ADG2 as an RFLP probe using restriction with EcoRI. The ADG2 was further tested on a total of 12 cultivars/genotypes of tomato (Lycopersicon), pepper (Capsicum), and eggplant (Solanum) (Table 4).

Potato breeding lines and cultivars were maintained as in vitro cultures on Murashige and Skoog medium (Murashige and Skoog 1962) at pH 5.8. Seeds of the cultivars/genotypes of other solanaceous crops were planted to and grown in soil (Perlite: Vermiculite : Peatmoss $=1: 1: 2$ ).

\subsection{DNA preparation}

Total DNA for RFLP analysis was extracted with hexadecyl trimethylammonium bromide (CTAB) method with some modification as described by Landry (1993). Total DNAs were digested with restriction enzyme EcoRI (Boehringer Mannheim, Germany) according to the manufacturer's instruction, and $2 \mu \mathrm{g}$ of the digested DNA was loaded and separated on $0.8 \%$ agarose gel using electrophoresis. Southern blotting was carried out as described by Sambrook et al. (1989).

\subsection{PCR amplification of ADG2 fragment}

The ADG2 was amplified by PCR from the total DNA of diploid potato breeding line $2 x(V-2)_{7}$ carrying $R y_{a d g}$ as previously described (Hämäläinen et al. 1998). The amplified fragment (355 bp) was cloned in pGEM - ${ }^{\circledR}$ Easy Vector (Promega co., USA). The clone was digested with EcoRI and electrophoresed in $2 \%$ agarose gel to separate the inserted DNA and the vector. The inserted DNA was extracted from agarose gel using a QIAquick ${ }^{\mathrm{TM}}$ Gel Extraction Kit (QIAGEN GmbH, Germany) to use for the RFLP probe. 
Table 1. Examination of potato cultivars and breeding lines and other solanaceous species tested with ADG2 as an RFLP marker

\begin{tabular}{|c|c|c|c|c|c|c|}
\hline \multirow{2}{*}{ Potato Clone } & \multirow{2}{*}{ Ploidy } & \multirow{2}{*}{$\begin{array}{c}\text { Resistance } \\
\text { donor } \\
\text { species }^{1}\end{array}$} & \multirow{2}{*}{$\begin{array}{l}\text { Resistance } \\
\text { phenotype }^{2}\end{array}$} & \multicolumn{2}{|c|}{$\begin{array}{c}\text { Presence of } \\
\text { marker band }\end{array}$} & \multirow[t]{2}{*}{ References } \\
\hline & & & & $10.5 \mathrm{~kb}$ & $3.5 \mathrm{~kb}$ & \\
\hline $2 x(V-2)_{7}$ & $2 \mathrm{x}$ & $a d g$ & $\mathrm{E}$ & + & + & Valkonen et al. 1994a, Watanabe et al. 1994a \\
\hline$\left\{2 \mathrm{x}(\mathrm{V}-2)_{7} \times 84.194 .30\right\}_{62}$ & $2 \mathrm{x}$ & $a d g$ & $\mathrm{E}$ & + & + & Sorri et al. 1999 \\
\hline 84.194 .30 & $2 \mathrm{x}$ & & $\mathrm{S}$ & - & - & Valkonen et al. 1994a, Watanabe et al. 1994a \\
\hline $7 X Y .1$ & $4 x$ & adg & $\mathrm{E}$ & + & + & Iwanaga et al. 1991, Watanabe et al. 1994b \\
\hline 84.35 .7 & $2 \mathrm{x}$ & & $\mathrm{S}$ & - & - & Watanabe et al. $1994 \mathrm{a}$ \\
\hline 84.36 .29 & $2 \mathrm{x}$ & & $\mathrm{S}$ & - & - & Watanabe et al. 1994a \\
\hline 85.37 .38 & $2 \mathrm{x}$ & & $\mathrm{S}$ & - & - & Watanabe et al. 1994a \\
\hline 86.54 .18 & $4 \mathrm{x}$ & sto & $\mathrm{E}^{*}$ & + & - & CIP $^{4} 1998$ \\
\hline 86.61 .26 & $2 \mathrm{x}$ & sto & $\mathrm{E}^{*}$ & + & - & Valkonen et al. 1994a, Watanabe et al. 1994a \\
\hline 87HW13.7 & $2 \mathrm{x}$ & & $\mathrm{S}$ & - & - & Valkonen et al. 1995 \\
\hline 90.30 .47 & $2 \mathrm{x}$ & & $S$ & - & - & E. Fernandez-Northcote, personal communication \\
\hline 90.31 .42 & $2 \mathrm{x}$ & & $\mathrm{S}$ & - & - & E. Fernandez-Northcote, personal communication \\
\hline $954.3 \mathrm{CA}$ & $4 \mathrm{x}$ & & $S$ & - & - & Watanabe et al. $1994 \mathrm{~b}$ \\
\hline $\mathrm{AA}-3$ & $4 \mathrm{x}$ & adg & $\mathrm{E}$ & + & + & Iwanaga et al. 1991, Watanabe et al. 1994b \\
\hline $\operatorname{acl} 7-8$ & $4 \mathrm{x}$ & & $\mathrm{S}$ & - & - & S. Slack, personal communication \\
\hline BW5.116 & $4 x$ & & $\mathrm{~S}$ & - & - & E. Fernandez-Northcote, personal communication \\
\hline СРC2451 & $2 \mathrm{x}$ & brd & $\mathrm{E}^{*}$ & - & - & Valkonen et al. 1995, Watanabe et al. $1995 \mathrm{~b}$ \\
\hline DG81- 68 & $2 \mathrm{x}$ & & S & - & - & Swiezynski et al. 1989 \\
\hline E74-7 & $4 x$ & $a d g$ & $\mathrm{E}$ & + & + & Hämäläinen et al. 1997 \\
\hline$F_{1}-1$ & $2 \mathrm{x}$ & chc & $\mathrm{E}^{*}$ & + & - & Hosaka and Hanneman 1994 \\
\hline $\mathrm{HHI}-9.3 \mathrm{CD}$ & $4 \mathrm{x}$ & & S & - & - & E. Fernandez-Northcote, personal communication \\
\hline I12.1 & $4 \mathrm{x}$ & & $\mathrm{S}$ & + & - & E. Fernandez-Northcote, personal communication \\
\hline IvP35 & $2 \mathrm{x}$ & phu & $\mathrm{E}^{*}$ & + & - & Valkonen et al. 1995 \\
\hline N140-201 & $4 x$ & $a d g$ & $\mathrm{E}$ & + & + & Hämäläinen et al. 1997 \\
\hline Q237-8 & $4 \mathrm{x}$ & adg & $\mathrm{E}$ & + & + & Hämäläinen et al. 1997 \\
\hline$S 48-6$ & $4 \mathrm{x}$ & & $\mathrm{H}$ & - & - & R. L. Plaisted, unpublished \\
\hline TA3.5.3.6 & $4 \mathrm{x}$ & $a d g$ & $\mathrm{E}$ & + & + & Watanabe et al. 1992 \\
\hline TA3.5.3.7 & $4 \mathrm{x}$ & $a d g$ & $\mathrm{E}$ & + & + & Watanabe et al. 1992 \\
\hline TA3.8.3.3 & $4 \mathrm{x}$ & $a d g$ & $\mathrm{E}$ & + & + & Watanabe et al. 1992 \\
\hline TET38.2 & $2 \mathrm{x}$ & & $\mathrm{H}$ & - & - & Valkonen et al. 1995, Watanabe et al. 1995b \\
\hline TET38.9 & $2 \mathrm{x}$ & & $\mathrm{E}^{*}$ & - & + & Valkonen et al. 1995, Watanabe et al. $1995 \mathrm{~b}$ \\
\hline TET38.12 & $2 \mathrm{x}$ & & $\mathrm{E}^{*}$ & - & - & Valkonen et al. 1995, Watanabe et al. 1995b \\
\hline TET38.13 & $2 \mathrm{x}$ & & $\mathrm{H}$ & - & - & Valkonen et al. 1995, Watanabe et al. 1995b \\
\hline A6 & $5 x$ & & $S$ & + & - & Russo and Slack 1998 \\
\hline All Blue & $4 x$ & & $S$ & + & - & CIP 1998 \\
\hline Alpha & $4 \mathrm{x}$ & & $\mathrm{S}$ & - & - & CIP 1998 \\
\hline Andover & $4 \mathrm{x}$ & & $\mathrm{S}$ & - & - & Plaisted et al. 1998 \\
\hline Arran Banner & $4 \mathrm{x}$ & & $S$ & + & - & A. Arihara, personal communication \\
\hline Astarte & $4 x$ & & S & - & - & HKAES $^{5} 1998$ \\
\hline Atzimba & $4 x$ & & $\mathrm{~S}$ or $\mathrm{H}$ & - & - & CIP 1998 \\
\hline Atlantic & $4 x$ & & S & - & - & Valkonen et al. 1994b, Russo and Slack 1998 \\
\hline Benimaru & $4 x$ & & $\mathrm{~S}$ & - & - & HKAES 1998 \\
\hline Bintje & $4 x$ & & $S$ & - & - & $\begin{array}{l}\text { Valkonen and Palohuhta 1996, Stegemann and } \\
\text { Schnick } 1982\end{array}$ \\
\hline Charles Downing & $4 x$ & & $\mathrm{~S}$ & - & - & A. Arihara, personal communication \\
\hline Chidiwa & $4 x$ & & $\mathrm{~S}$ & - & - & HKAES 1998 \\
\hline Chieftain & $4 x$ & & $S$ & - & - & CIP 1998 \\
\hline Cutetia & $4 x$ & & $\mathrm{~S}$ & - & - & A. Arihara, personal communication \\
\hline Dannsyaku & $4 x$ & & $\mathrm{~S}$ & + & + & HKAES 1998 \\
\hline Dejima & $4 x$ & & $\mathrm{~S}$ & - & - & HKAES 1998 \\
\hline Deodara & $4 x$ & & $\mathrm{~S}$ & - & - & HKAES 1998 \\
\hline Desiree & $4 x$ & $t b r$ & $\mathrm{H}$ & - & - & Jones 1990, Stegemann and Schnick 1982 \\
\hline Early Gem & $4 x$ & & S & - & - & A. Arihara, personal communication \\
\hline Eniwa & $4 x$ & & $\mathrm{~S}$ & - & - & HKAES 1998 \\
\hline Erd manner & $4 x$ & & $\mathrm{~S}$ & - & - & A. Arihara, personal communication \\
\hline Ezoakari & $4 x$ & & $\mathrm{~S}$ & - & - & HKAES 1998 \\
\hline
\end{tabular}


Table 1. continued

\begin{tabular}{|c|c|c|c|c|c|c|}
\hline \multirow[t]{2}{*}{ Potato Clone } & \multirow[t]{2}{*}{ Ploidy } & \multirow{2}{*}{$\begin{array}{l}\text { Resistance } \\
\text { donor } \\
\text { species }^{1}\end{array}$} & \multirow{2}{*}{$\begin{array}{l}\text { Resistance } \\
\text { phenotype }^{2}\end{array}$} & \multicolumn{2}{|c|}{$\begin{array}{l}\text { Presence of } \\
\text { marker band }\end{array}$} & \multirow[t]{2}{*}{ References } \\
\hline & & & & $10.5 \mathrm{~kb}$ & $3.5 \mathrm{~kb}$ & \\
\hline Fabricia & $4 x$ & & $\mathrm{~S}$ & - & - & HKAES 1998 \\
\hline Fina & $4 x$ & & $S$ & - & - & HKAES 1998 \\
\hline Firmula & $4 x$ & & S & - & - & A. Arihara, personal communication \\
\hline Furore & $4 x$ & & S & - & - & A. Arihara, personal communication \\
\hline Gabriela & $4 x$ & & S & - & - & A. Arihara, personal communication \\
\hline Gineke & $4 x$ & & S & - & - & CIP 1998 \\
\hline Greta & $4 x$ & & S & - & - & CIP 1998 \\
\hline Hatsufubuki & $4 x$ & & $S$ & - & - & HKAES 1998 \\
\hline Hokkaishiro & $4 x$ & & $S$ & - & - & HKAES 1998 \\
\hline Inka-no-Hoshi & $4 x$ & & $S$ & - & - & HKAES 1998 \\
\hline Jakko & $4 x$ & & $S$ & + & - & A. Arihara, personal communication \\
\hline King Edward & $4 x$ & & $\mathrm{~S}$ & - & - & Jones 1990, Stegemann and Schnick 1982 \\
\hline Kitaakari & $4 x$ & & S & - & - & HKAES 1998 \\
\hline Konafubuki & $4 x$ & chc & $\mathrm{E}^{*}$ & + & - & HKAES 1998 \\
\hline La Soda & $4 x$ & & S & - & - & A. Arihara, personal communication \\
\hline M. Hindenburg & $4 x$ & & $\mathrm{~S}$ & + & - & CIP 1998 \\
\hline Matilda & $4 x$ & & $\mathrm{~S}$ & - & - & Valkonen and Palohuhta 1996 \\
\hline May Queen & $4 x$ & & $\mathrm{~S}$ & - & - & HKAES 1998 \\
\hline Mentor & $4 x$ & & $\mathrm{~S}$ & + & - & CIP 1998 \\
\hline Musamaru & $4 x$ & & $S$ & - & - & HKAES 1998 \\
\hline Myojo & $4 \mathrm{x}$ & & $S$ & + & - & HKAES 1998 \\
\hline Norchip & $4 x$ & & $\mathrm{~S}$ & - & - & CIP 1998 \\
\hline NY99 & $4 x$ & & $\mathrm{~S}$ & + & + & Hämäläinen et al. 1997 \\
\hline NY103 & $4 x$ & $\operatorname{adg}$ & $\mathrm{E}$ & - & + & CIP 1998 \\
\hline NY109 & $4 x$ & & $\mathrm{~S}$ & + & - & CIP 1998 \\
\hline NY115 & $4 x$ & & $S$ & + & - & CIP 1998 \\
\hline NY121 & $4 x$ & $a d g$ & $\mathrm{E}$ & + & + & CIP 1998 \\
\hline NY123 & $4 x$ & $a d g$ & $\mathrm{E}$ & - & + & CIP 1998 \\
\hline Ohjiro & $4 x$ & & $\mathrm{~S}$ & - & - & HKAES 1998 \\
\hline P. Wohitman & $4 x$ & & $\mathrm{~S}$ & - & - & A. Arihara, personal communication \\
\hline Papa Amarilla & $2 x$ & & $S$ & - & - & CIP 1998 \\
\hline Panther & $4 x$ & & $\mathrm{~S}$ & - & - & A. Arihara, personal communication \\
\hline Parnassia & $4 x$ & & $\mathrm{~S}$ & - & - & A. Arihara, personal communication \\
\hline Pentland Ace & $4 \mathrm{x}$ & & $\mathrm{S}$ & - & - & CIP 1998 \\
\hline Pentland Crown & $4 x$ & & $\mathrm{H}$ & - & - & Jones 1990, Valkonen et al. $1994 \mathrm{~b}$ \\
\hline Pentland Dell & $4 \mathrm{x}$ & & $\mathrm{H}$ & + & - & $\begin{array}{l}\text { Stegemann and Schnick 1982, Valkonen et al. } \\
\text { 1994b, } 1998\end{array}$ \\
\hline Pentland Ivory & $4 \mathrm{x}$ & $t b r$ & $\mathrm{H}$ & - & - & Jones 1990, Stegemann and Schnick 1982 \\
\hline Pito & $4 x$ & $t b r$ & $\mathrm{H}$ & - & - & Valkonen 1997, Stegemann and Schnick 1982 \\
\hline Pukara & $4 \mathrm{x}$ & & S & + & - & A. Arihara, personal communication \\
\hline Pungo & $4 x$ & & $\mathrm{~S}$ & - & - & A. Arihara, personal communication \\
\hline Purple Peruvian & $4 \mathrm{x}$ & & $S$ & + & - & CIP 1998 \\
\hline Puskin & $4 x$ & & $S$ & - & - & A. Arihara, personal communication \\
\hline Rishiri & $4 x$ & & $S$ & - & + & HKAES 1998 \\
\hline Red Andes & $4 x$ & & $S$ & - & - & A. Arihara, personal communication \\
\hline Russet Burbank & $4 x$ & & $\mathrm{~S}$ & - & - & CIP 1998 \\
\hline Russet Rural & $4 x$ & & $\mathrm{~S}$ & - & - & CIP 1998 \\
\hline Sakurafubuki & $4 x$ & chc & $\mathrm{E}^{*}$ & + & + & HKAES 1998 \\
\hline Sequoia & $4 x$ & & $S$ & - & - & A. Arihara, personal communication \\
\hline Serrana & $4 x$ & & $\mathrm{H}$ & - & - & CIP 1998 \\
\hline Shimabara & $4 \mathrm{x}$ & & $S$ & - & - & HKAES 1998 \\
\hline Shiretoko & $4 x$ & & $\mathrm{~S}$ & - & - & HKAES 1998 \\
\hline Snowden & $4 x$ & & $\mathrm{~S}$ & - & - & CIP 1998 \\
\hline Stirling & $4 x$ & & $\mathrm{~S}$ & - & - & CIP 1998 \\
\hline Touya & $4 x$ & & $\mathrm{~S}$ & - & - & HKAES 1998 \\
\hline Toyoakari & $4 x$ & & $S$ & - & - & HKAES 1998 \\
\hline
\end{tabular}


Table 1. continued

\begin{tabular}{|c|c|c|c|c|c|c|}
\hline \multirow{2}{*}{ Potato Clone } & \multirow{2}{*}{ Ploidy } & \multirow{2}{*}{$\begin{array}{c}\text { Resistance } \\
\text { donor } \\
\text { species }^{1}\end{array}$} & \multirow{2}{*}{$\begin{array}{l}\text { Resistance } \\
\text { phenotype }^{2}\end{array}$} & \multicolumn{2}{|c|}{$\begin{array}{l}\text { Presence of } \\
\text { marler band }\end{array}$} & \multirow{2}{*}{ References } \\
\hline & & & & $10.5 \mathrm{~kb}$ & $3.5 \mathrm{~kb}$ & \\
\hline Toyoshiro & $4 \mathrm{x}$ & & $\mathrm{S}$ & - & - & HKAES 1998 \\
\hline Tsunika & $4 \mathrm{x}$ & & $\mathrm{S}$ & - & - & HKAES 1998 \\
\hline Waseshiro & $4 \mathrm{x}$ & & $\mathrm{S}$ & - & - & HKAES 1998 \\
\hline Yagana & $4 \mathrm{x}$ & & $\mathrm{S}$ & - & - & CIP 1998 \\
\hline Yellow Fin & $4 \mathrm{x}$ & & S & - & - & S. Slack, personal communication \\
\hline Yukijiro & $4 \mathrm{x}$ & & $\mathrm{S}$ & - & - & HKAES 1998 \\
\hline Yukon Gold & $4 \mathrm{x}$ & & $\mathrm{H}$ & - & - & Valkonen 1997 \\
\hline
\end{tabular}

${ }^{1}$ adg: Solanum tuberosum subsp. andigena, brd: S. brevidens, chc: S. chacoense, sto: S. stoloniferum, phu: S. phureja, tbr: S. tuberosum

${ }^{2}$ E, extreme resistant by $R y_{a d g}: \mathrm{E}^{*}$, extreme resistant by different genes from $R y_{\text {adg }} ; \mathrm{H}$, hypersensitive; $\mathrm{S}$, susceptible

${ }^{3}$ Marker band detected $(+)$ or not detected $(-)$

${ }^{4}$ International Potato Center

${ }^{5}$ Hokkaido Konsen Agricultural Experimental Station, Japan

\subsection{RFLP analysis}

Hybridization was done overnight at $65{ }^{\circ} \mathrm{C}$. The membranes were washed with the stringency wash buffer ( $2 \mathrm{M}$ Urea, $0.1 \%$ SDS, $50 \mathrm{mM}$ Na phosphate, $150 \mathrm{mM} \mathrm{NaCl}, 10 \mathrm{mM} \mathrm{MgCl}_{2}$ ). The first wash was carried out at $55{ }^{\circ} \mathrm{C}$ for $5 \mathrm{~min}$, and the second wash was carried out at $58{ }^{\circ} \mathrm{C}$ for $5 \mathrm{~min}$. Chemiluminescent detection of the probe using AlkPhos Direct ${ }^{\mathrm{TM}}$ non-radioactive system and Hyperfilm $^{\mathrm{TM}}$ (Amersham Pharmacia, UK) was carried out according the manufacturer's instructions.

\section{Results and Discussion}

A new signals specific to the $\mathrm{E}$ phenotype was observed at $10.5 \mathrm{~kb}$ among the many bands detected in the tested potato genotypes (Fig. 1, Table 1). It has already been reported that the $3.5 \mathrm{~kb}$ band cosegregated with $R y_{a d g}$ in a mapping population consisted of 77 progeny (Hämäläinen et al. 1998). The $10.5 \mathrm{~kb}$ signal was newly recognized to be associated with $R y_{\text {adg }}$ in this study.

Applicability test for the $3.5 \mathrm{~kb}$ marker using various potato genotypes revealed that the $3.5 \mathrm{~kb}$ marker was specific to genotypes carrying $R y_{a d g}$ (Table 1). The $3.5 \mathrm{~kb}$ signal was detected in 13 of 13 genotypes carrying $R y_{a d g}$, but not detected at 99 of 104 genotypes lacking this gene. The association between the $3.5 \mathrm{~kb}$ signal and the $R y_{a d g}$ was $96 \%$ (Table 2). Thus, it was confirmed that the $3.5 \mathrm{~kb}$ marker can be used specifically for the selection on the $R y_{a d g}$ gene, regardless of the diverse genetic background in materials, as previously suggested (Hämäläinen et al. 1998).

On the other hand, the $10.5 \mathrm{~kb}$ signal was also

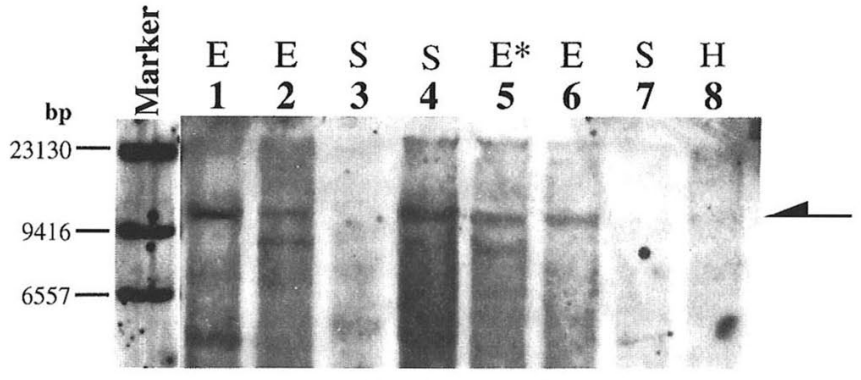

Fig. 1 Southern hybridization with the ADG2 to EcoRI-digested genomic DNA of diploid and tetraploid potato breeding lines and tetraploid potato cultivars which are extremely resistant (E), susceptible (S) to PVY. Lane 1: $2 \mathrm{x}(\mathrm{V}-2)_{7}(\mathrm{E}), 2$ : $\left\{2 x(V-2)_{7} \times 84.194 .30\right\}_{62}(\mathrm{E}), 3: 84.194 .30$ (S), 4: NY115(S), 5: IvP35 (E*), 6: NY121(E), 7: Stirling (S), 8: Pito (H). Marker: lambda DNA Hind III digested. The E phenotype in IvP35 is supposed to be controlled by a resistance gene derived from S. phureja.

detected in the E phenotypes supposed to be controlled by resistance genes derived from $S$. phureja (IvP35), $S$. chacoense $\left(\mathrm{F}_{1}-1\right.$, Konafubuki, and Sakurafubuki), and $S$. stoloniferum (86.54.18 and 86.61.26) (Watanabe et al. 1994a) in addition to genotypes carrying $R y_{a d g}$, (Table 1). This signal was detected in 17 of 22 genotype carrying $R y_{a d g}$ and other $R y$ genes, and the association between the $10.5 \mathrm{~kb}$ signal and the phenotypes was $83 \%$ (Table 3). Therefore, the $10.5 \mathrm{~kb}$ marker could select $\mathrm{E}$ phenotypes in potato cultivars and lines with various genetic backgrounds. This is the complementary function of $10.5 \mathrm{~kb}$ signals to $3.5 \mathrm{~kb}$ signals which specifically linked to $R y_{a d g}$.

As for some potato clones with $\mathrm{E}$ phenotype such 
Table 2. Correspondence between ADG2/ Eco RI signals and $R y_{\text {adg }}$

\begin{tabular}{ccc}
\hline & $10.5 \mathrm{~kb} \mathrm{signal}^{1}$ & $3.5 \mathrm{~kb}$ signal \\
\hline $\begin{array}{c}R y_{\text {adg }} \\
\text { other } R y\end{array}$ & $11 / 13$ & $13 / 13$ \\
$\mathrm{~S}$ and $\mathrm{H}^{2}$ & $6 / 9$ & $2 / 9$ \\
\hline Association $^{3}$ & $15 / 95$ & $3 / 95$ \\
\hline${ }^{1}$ a denominator: total number of tested genotpe, a numerator. total of genetype detected signal \\
${ }^{2} \mathrm{~S}:$ susceptible, H; hypersesitive \\
${ }^{3}$ Correlations between $R y_{\text {adg }}$ and marker- signal were calculated as follow; A numenrator is \\
sum of the number of the line exhibited signal in genotypes carrying $R y_{\text {adg }}$ and the number of \\
line lacking marker - signal in $\mathrm{S}, \mathrm{H}$ and other $R y$. A denominator is sum of number of tested \\
lines.
\end{tabular}

Table 3. Correspondence between ADG2/Eco RI signals and PVY resistance phenotype.

\begin{tabular}{lcc}
\hline PVY resistant phenotype $^{1}$ & 10.5kb signal & \\
\hline $\mathrm{E}$ & $17 / 22$ & $3.5 \mathrm{~kb} \mathrm{signal}^{2}$ \\
$\mathrm{~S}$ and H & $15 / 95$ & $15 / 22$ \\
& $97 / 117=83 \%$ & $107 / 117=91 \%$ \\
\hline Association & \\
\hline${ }^{1} \mathrm{E}:$ Extreme resistance, S: susceptible, $\mathrm{H}:$ hypersensitive & \\
${ }^{2}$ a denominator; total of tested phenotype, a numerator: total of phenotype detected signal \\
${ }^{3}$ Correlations between PVY resistant phenotype and marker- signal were calculated as follow: \\
A numenrator is sum of the number of the line exhibited signal in E phenotype and the \\
number of line lacking marker- signal in S and H. A denominator is sum of number of tested \\
lines.
\end{tabular}

as CPC2451 and TET38.12, which did not show the positive signals, suggestions shall be referred to Watanabe et al. (1995b) and (Valkonen et al. 1995). Distantly related species (CPC2451, S. brevidens) and its interspecific hybrid (TET38.12) with a diploid potato breeding line Watanabe et al. (1995b), could have alternative genetics and resistance gene(s) conferring E phenotype (Valkonen et al. 1995). It should also be noted that there were some cultivars with $\mathrm{S}$ phenotype such as Dansyaku and NY99, which showed the positive signals. While they are exception in the percentage (Tables 2 and 3), extra caution shall be taken when these genotypes and progenies are to be used for MAS with the markers discussed in this paper. Although ADG2-EcoRI RFLP fragments have high association for the distinction of the $\mathrm{E}$ phenotypes with PVY resistance, the ADG2 fragment may not be the part of an $R y$ gene. It may be adjacent to an $R y$ locus but with a distance which allows recombination as derivatives such as Dansyaku and NY99 exist, or it is independent with a similar motif like an $R y$ gene (Watterson et al. 1993).

An application of markers that is reliable and easy to use is preferable in practical use which includes a large number of samples and time-consuming steps. Obtaining two indexes from one procedure is a very attractive feature of the RFLP marker ADG2, even though some optimizations of the hybridization condition will be required. Moreover, the intriguing findings in this report should be addressed here; MAS using the marker ADG2/EcoRI can be carried out on a relatively wide range of resistance sources based on the $10.5 \mathrm{~kb}$ marker, whereas specific selection for the $R y_{a d g}$ can be done with the $3.5 \mathrm{~kb}$ marker. Thereafter, our survey results clearly indicate that the RFLP marker ADG2 provides a powerful tool in breeding of PVY resistant potato cultivars.

As the ADG2 as an RFLP probe was applied to EcoRIdigested genomic DNA of tomato (Lycopersicon), pepper (Capsicum), and eggplant (Solanum), which are representative species within the family Solanaceae and susceptible to PVY, signals were detected in at least one line of each of the tested species (Table 4). Twelve signals with different sizes were detected in these lines. In 'Fox Face' (eggplant), only one signal was detected, whereas in 
Table 4. Distribution of signals detected by ADG2

\begin{tabular}{|c|c|c|c|c|c|c|c|c|c|c|c|c|}
\hline \multirow[t]{2}{*}{ Material name } & \multirow{2}{*}{$\begin{array}{l}\text { No. of } \\
\text { signal }\end{array}$} & \multicolumn{11}{|c|}{ Detected signals ${ }^{1}$} \\
\hline & & 2.5 & 3.0 & $3.5^{2}$ & 4.0 & 4.5 & 5.0 & 5.5 & 7.0 & 7.5 & 9.0 & $10.5^{2}(\mathrm{~kb})$ \\
\hline \multicolumn{13}{|l|}{ Lycopersicon } \\
\hline Rejina & 3 & - & - & + & - & - & + & - & - & - & - & + \\
\hline Ogata-tomato & 4 & + & - & + & - & - & + & - & - & - & - & + \\
\hline Sugar Lamp & 7 & + & - & + & - & - & + & + & - & + & + & + \\
\hline Home-Momotaro & 3 & + & + & - & + & - & - & - & - & - & - & - \\
\hline Pepe & 2 & + & + & - & - & - & - & - & - & - & - & - \\
\hline Yellow Cherry & 2 & - & + & - & - & - & + & - & - & - & - & - \\
\hline \multicolumn{13}{|l|}{ Capsicum } \\
\hline Ornamental Capsicum & 0 & - & - & - & - & - & - & - & - & - & - & - \\
\hline Fushimi- Amanaga & 0 & - & - & - & - & - & - & - & - & - & - & - \\
\hline Shishito & 3 & + & - & - & - & + & - & + & - & - & - & - \\
\hline Kyomidori & 2 & - & + & - & - & - & + & - & - & - & - & - \\
\hline \multicolumn{13}{|l|}{ Solanum } \\
\hline Fox Face & 1 & - & - & - & - & - & - & - & + & - & - & - \\
\hline Bay-nasu & 0 & - & - & - & - & - & - & - & - & - & - & - \\
\hline
\end{tabular}

${ }^{1}+$ : detected, $-:$ not detected

${ }^{2} 3.5 \mathrm{~kb}$ and $10.5 \mathrm{~kb}$ signals were showed to be linked to PVY resistance in potatoes.

'Sugar Lamp' (tomato) seven signals were detected. Other genotypes had from two to six signals. Presence of these signals suggested ADG2 homologous regions are also present in solanaceous species. Interestingly, the $3.5 \mathrm{~kb}$ signal and the $10.5 \mathrm{~kb}$ signal, which were linked to PVY resistance in potato, were also detected in some lines of tomato.

RFLP linkage maps are now available for many plant species, including three important members of the Solanaceae, potato, tomato, and pepper, and indicate similarities of the genomes between potatoes and tomatoes (Gebhardt et al. 1991, Prince et al. 1993, Tanksley et al. 1992). Indeed, some RFLP markers derived from tomato genomic DNA or cDNA are linked to PVY resistance genes in potatoes (Hämäläinen et al. 1997). Thus, the ADG2 may be useful for searching PVY resistance in the nontuber bearing species within the family Solanaceae. This needs to be tested in a future study.

\section{Acknowledgments}

DNA samples of potato cultivars were provided from CIP, Cornell University and Hokuren Federation of Agricultural Cooperatives. We thank Dr. Kazuyoshi Hosaka, Exp. Stn., Fac. Agr., Kobe Univ. for his helpful advice and for providing some plant materials for conducting this experiment. We are grateful to Yasuhiko Takeda, Youko Morikawa and Junko Kyotani for technical assistance. This research was supported by the grant JSPS RFTF96L00603 to KNW and KK, and was partially supported by a grant-in-aid No. 09760007 from the Ministry of Education, Science, Sports and Culture, Japan, and by the Academy of Finland (grant \#36256).

\section{References}

Brigneti, G., Garcia-Mas, J., Baulcombe, D. C., 1997. Molecular mapping of the potato virus $\mathrm{Y}$ resistance gene $R y_{\text {sto }}$ in potato. Theor. Appl. Genet., 94: 198- 203.

CIP, 1998. List of Pathogen Tested Potato Genotypes. International Potato Center, Lima, Peru.

Cockerham, G., 1970. Genetical studies on resistance to potato viruses X and Y. Heredity, 25: 309-348.

de Bokx, J. A., Huttinga, H., 1981. Potato virus Y. CMI/AAB Descriptions of Plant Viruses no. 242.

de Bokx, J. A., van der Want, J. P. H., 1987. Viruses of potatoes and seed-potato production. PUDOC, Wageningen, The Netherlands.

Gebhardt, C., Ritter, E., Barone, A., Debener, T., Walkemeier, B., Schachtschabel, U., Kaufmann, H., Thompson, R. D., Bonierbale, M. W., Ganal, M. W., Tanksley, S. D., Salamini, F., 1991. RFLP maps of potato and their alignment with the homoeologous tomato genome. Theor. Appl. Genet., 83: 49- 57.

Hawkes, J. G., 1990. The Potato. Evolution, Biodiversity and Genetic Resources. Smithsonian Institution, Washington, D. C.

Hämäläinen, J. H., Watanabe, K. N., Valkonen, J. P. T., Arihara, A., Plaisted, R. L., Pehu, E., Miller, L., Slack, S. A., 1997. Mapping and marker-assisted selection for a gene for extreme resistance to potato virus $Y$. Theor. Appl. Genet., 94: 192-197.

Hämäläinen, J. H., Sorri, V. A., Watanabe, K. N., Gebhardt, 
C., Valkonen, J. P. T., 1998. Molecular examination of a chromosome region that controls resistance to potato $\mathrm{Y}$ and A potyviruses in potato. Theor. Appl. Genet., 96: 1036-1043.

HKAES, 1998. Report on Potato Breeding 1996. Hokkaido Konsen Agricultural Experimental Station, Japan. (In Japanese)

Hooker, W. J., 1981. Compendium of Potato Diseases. APS Press, St. Paul, Minnesota.

Hosaka, K., Hanneman, R. E., Jr., 1994. Random amplified polymorphic DNA markers detected in a segregating hybrid population of Solanum chacoense X S. phureja. Jpn. J. Genet., 69: 53-66.

Iwanaga, M., Freyre, R., Watanabe, K., 1991. Breaking the crossabillity barriers between disomic tetraploid Solanum acaule and tetrasomic tetraploid $S$. tuberosum. Euphytica, 52: 183-191.

Jones, R. A. C., 1990. Strain grope specific and virus specific hypersensitive reactions to infection with potyviruses in potato cultivars. Ann. Appl. Biol., 117: 93105.

Kasai, K., Morikawa, Y., Sorri, V. A., Valkonen, J. P. T., Gebhardt, C., Watanabe, K., 1999. Development of SCAR markers to the PVY resistance gene $R y_{\text {adg }}$ based on a common feature of plant disease resistance genes. Genome Accepted.

Landry, B. S., 1993. DNA Mapping in Plants, In: Glick, B.R. Thompson, J.E. (Eds): Methods in Plant Molecular Biology and Biotechnology, 267 - 285. CRC Press, Florida.

Leister, D., Ballvora, A., Salamini, F., Gebhardt, C., 1996. A PCR - based approach for isolating pathogen resistance genes from potato with potential for wide application in plants. Nature Genetics, 14: 421-429.

Murashige, T., Skoog, F., 1962. A revised medium for rapid growth and bioassays with tobacco tissue culture. Physiol. Plant., 15: 473-497.

Plaisted, R. L., Halseth, D. E., Brodie, B. B., Slack, S. A., Sieczka, J. B., Christ, B. J., Paddock, K. M., Peck, M. W., 1998. Andover: An early to midseason golden nematode resistant variety for use as chipstock or tablestock. Amer. J. Potato Res., 75: 113- 116.

Prince, J. P., Pochard, E., Tanksley, S. D., 1993. Construction of a molecular linkage map of pepper and a comparison of synteny with tomato. Genome, 36: 404417.

Ross, H., 1986. Potato breeding - Problems and Perspectives. J. Plant Breed. Suppl, 13: 132.

Russo, P., Slack, S. A., 1998. Tissue culture methods for the screening and analysis if putative virus-resistant transgenic potato plants. Virology, 88: 437-441.

Sambrook, J., Fritsch, E. F., Maniatis, T., 1989. Molecular cloning: a laboratory manual, 2nd edn. Cold Spring Harbor Laboratory Press, Plainview, New York.

Shukla, D. D., Ward, C. W., Brunt, A. A., 1994. The Potyviridae. CAB International, Wallingford.

Sorri, V. A., Watanabe, K. N., Valkonen, J. P. T., 1999. Predicted kinase $3 \mathrm{a}$ motif of a resistance gene analogue as a unique marker for virus resistance. Theor. Appl.
Genet., 99: 164- 170 .

Stegmann, H., Schick, D., 1982. Index 1982 of European potato varieties. Biologische Bundesanstait fur Landund Forstwirtschaht, Institut fur Biochemie, Braunschweig, Berlin.

Swiezynski, K. M., Dziewonska, M. A., Ostrowska, K., 1989. Resistance to the potato leafroll virus (PLRV) in diploid potatoes. Plant Breed., 103: 221- 227.

Tanksley, S. D., Ganal, M. W., Prince, J. P., Vicente, M. C. d., Bonierbale, M. W., Broun, P., Fulton, T. M., Giovannoni, J. J., Pineda, O., R_der, M. S., Wing, R. A., Wu, W., Young, N. D., 1992. High density molecular linkage maps of the tomato and potato genomes. Genetics, 132: 1141-1160.

Valkonen, J. P. T., Slack, S. A., Plaisted, R. L., Watanabe, K. N., 1994a. Extreme resistance is epistatic to hypersensitive resistance to potato virus $\mathrm{Y}^{0}$ in a Solanum tuberosum subsp. andigena-derived potato genotype. Plant Disease, 78: 1177-1180.

Valkonen, J. P. T., Slack, S. A., Plaisted, R. L., 1994b. Use of the virus strain group concept to characterize the resistance to PVX and PVYo in the potato cv "Allegany". Am. Potato J., 71: 507-516.

Valkonen, J. P. T., Orrillo, M., Slack, S. A., Plaisted, R. L., Watanabe, K. N., 1995. Resistance to viruses in $F_{1}$ hybrids produced by direct crossing between diploid Solanum series Tuberosa and diploid $S$. brevidens (series Etuberosa) using $S$. phureja for rescue pollination. Plant Breed., 114: 421-426.

Valkonen, J. P. T., Jones, R. A. C., Slack, S. A., Watanabe, K. N., 1996. Resistance specificities to virus in potato: Standardization of nomenclature. Plant Breed., 115: 433-438.

Valkonen, J. P. T., Palohuhta, J. P., 1996. Resistance to potato virus $\mathrm{A}$ and Potato virus $\mathrm{Y}$ in potato cultivars grown in Finland. Agricultural and Food Science in Finland., 5: 57-62.

Valkonen, J. P. T., 1997. Novel resistance to four potyviruses in tuber-bearing potato species, and temperaturesensitive expression of hypersensitive resistance to potato virus Y. Ann. Appl. Biol., 130: 91-104.

Valkonen, J. P. T., Rokka, V.-M., 1998. Combination and expression of two virus resistance mechanisms in interspecific somatic hybrids of potato. Plant Science, 131: $85-94$.

Watanabe, K., Vega, S., Orrillo, M., 1992. Characterization on $S$. acaule introgression lines. Am. Potato J., 69: 613614.

Watanabe, K., Orrillo, M., Iwanaga, M., Ortiz, R., Freyre, R., Perez, S., 1994a. Diploid potato germplasm derived from wild and land race genetic resources. Am. Potato J., 71: 599-604.

Watanabe, K., Orrillo, M., Vega, S., Masuelli, R., Ishiki, K., 1994b. Potato germplasm enhancement with disomic tetraploid Solanum acaule. II. Assessment of breeding value of tetraploid $\mathrm{F}_{1}$ hybrids between tetrasomic tetraploid $S$. tuberosum and $S$. acaule. Theor. Appl. Genet., 88: $135-140$.

Watanabe, K. N., Orrillo, M., Golmirzaie, A. M., 1995 a. 
Potato germplasm enhancement for resistance to biotic stresses at CIP. Conventional and biotechnology - assisted approaches using a wide range of Solanum species. Euphytica, 85: 457-464.

Watanabe, K. N., Orrillo, M., Vega, S., Valkonen, J. P. T., Pehu, E., Hurtado, A., and Tanksley, S. D., 1995 b. Overcoming crossing barriers between nontuber-bear- ing and tuber-bearing Solanum species:towards potato germplasm enhancement with a broad spectrum of solanaceaous genetic resources. Genome, 38: 27-35.

Watterson, J. C., 1993. Development and breeding for resistance to pepper and tomato viruses. In: Kyle, M. M. (Ed.): Resistance to Viral Diseases of Vegetables, 80-101. Timber Press, Portland. 